\title{
The Functional Difference of Dendritic Cells in HBeAg Negative Chronic Hepatitis B Patients with Three Different Spleen Deficiency Syndromes and the Therapeutic Evaluation of Chinese Medicine Intervention Based on Syndrome Differentiation
}

\author{
Lei Wang, ${ }^{1,2}$ Li Zhang, ${ }^{1}$ Xiaoxia Feng, ${ }^{1}$ Lianjun Xing, ${ }^{1,2}$ Wei Zhang, ${ }^{2}$ Kaiping Jiang, \\ Haiyan Song, ${ }^{1}$ and Guang Ji ${ }^{1,4}$ \\ ${ }^{1}$ Institute of Digestive Diseases, Longhua Hospital, Shanghai University of Traditional Chinese Medicine, Shanghai 200032, China \\ ${ }^{2}$ Department of Hepatology, Longhua Hospital, Shanghai University of Traditional Chinese Medicine, Shanghai 200032, China \\ ${ }^{3}$ Department of Hepatology, Foshan Hospital of Traditional Chinese Medicine, Guangdong 528000, China \\ ${ }^{4}$ E-Institute of Shanghai Municipal Education Commission, Shanghai University of Traditional Chinese Medicine, \\ Shanghai 201203, China \\ Correspondence should be addressed to Haiyan Song; songhy@126.com and Guang Ji; jiliver@vip.sina.com
}

Received 12 February 2014; Revised 8 May 2014; Accepted 28 May 2014; Published 29 June 2014

Academic Editor: Charlie C. L. Xue

Copyright @ 2014 Lei Wang et al. This is an open access article distributed under the Creative Commons Attribution License, which permits unrestricted use, distribution, and reproduction in any medium, provided the original work is properly cited.

\begin{abstract}
Objective. To investigate the dendritic cells (DCs) maturity differences of HBeAg negative chronic hepatitis B (CHB) patients with different spleen deficiency (SD) syndromes and explore the role of syndrome differentiation in the therapeutic evaluation of Chinese medicine. Methods. 120 participants were recruited including three treatment groups in different SD syndrome categories as spleen deficiency with liver depression (SDLD), spleen deficiency with damp heat (SDDH), and spleen deficiency with kidney deficiency (SDKD) and one healthy control group; each group had 30 participants. Corresponding drugs were applied. The outcome measures included DC phenotype, liver function, IL-10, IL-12, and HBV-DNA levels. Results. The surface markers of mature DCs and cytokines levels were different in each group; the positive rate of CD80, CDla, HLA-DR, and CD1a was the lowest in SDKD group. After 3-month intervention, the expression of CD80, CD86, CDla, HLA-DR, and IL-12 significantly increased, while ALT, AST, and IL-10 significantly decreased $(P<0.05)$ in treatment groups. HBV-DNA level also significantly reduced in both SDKD and SDLD groups $(P<0.05)$. Conclusions. HBeAg negative patients had DCs dysmaturity, and there were differences between different SD syndromes. Chinese medicine intervention according to syndrome differentiation could advance the maturity and function of DCs and improve the therapeutic effect.
\end{abstract}

\section{Introduction}

Chronic hepatitis B (CHB) is a common infectious disease with high prevalence in the world, and about one million people die from hepatitis B virus (HBV) related hepatic failure, liver cirrhosis, and primary carcinoma annually $[1,2]$. Other than HBV infection, immune deficiency of the host is also the vital reason for the continuous existence of hepatitis $B$ virus $[3,4]$. Numerous studies have shown that there are various degrees of immune tolerance in $\mathrm{CHB}$ patients. Dendritic cells (DCs) are the most powerful antigen presenting cells (APC) in human, and their maturity and function have direct influence on cellular and humoral immunity [5-7]. Besides the immune characteristic of $\mathrm{CHB}, \mathrm{HBeAg}$ negative $\mathrm{CHB}$ also has the characteristics of low rate of antiviral treatment response, poor long-term prognosis, and high occurrence of hepatocellular carcinoma (HCC), which make the effects of conventional medical treatment unsatisfactory 
and make people seek for traditional Chinese medicine (TCM) to manage their symptoms [8].

According to TCM theory, syndrome differentiation is the critical step of disease diagnosis and treatment. In TCM, the spectrum of syndrome includes clinical information such as symptoms, signs, coating on the tongue, pulse condition, and the process to perceive this information. The prescriptions of the same disease may be different due to different syndromes. Studies have shown that the same disease with different TCM syndromes may have different substrates, and these differences might be helpful for exploring the Chinese syndrome classification and guiding the clinical treatment and therapeutic evaluation $[9,10]$. Spleen deficiency (SD) is the most common syndrome for CHB patients. However, the syndromes of $\mathrm{CHB}$ patients are not always the same, and most patients' clinical presentation may combine with other syndromes such as "liver depression," "damp heat," and "kidney deficiency." Researches of relationship between various syndrome phenotypes and disease characteristics will not only contribute to explain the scientific nature of Chinese syndrome classification, but also guide the strategy setting of the treatment and thus improve the clinical effects of Chinese treatment. According to the previous studies, SD is closely associated with immune dysfunction [11] and as DCs have the immunological characteristics, we hypothesized that there may be some differences of DC phenotype and function among various $\mathrm{SD}$ categories in $\mathrm{HBeAg}$ negative $\mathrm{CHB}$ patients, and these differences may influence the clinical effects of Chinese medicine treatment.

This study aims to assess the DC phenotype and function in different SD categories as well as other immune indexes (IL-10 and IL-12) among the HBeAg negative CHB patients based on the main therapy strategy of "treatment according to the syndrome." We hope it will be helpful to explain the immunological characteristics of Chinese medicine syndrome and shed light on the scientific mechanism of syndrome differentiation to improve the effects of Chinese medicine interventions.

\section{Materials and Methods}

2.1. Study Design. This study applies a prospective cohort control study method, and, according to TCM criteria, $\mathrm{HBeAg}$ negative $\mathrm{CHB}$ patients with spleen deficiency are divided into SDLD group (group A), SDDH group (group B), and spleen SDKD group (group C) and the normal control group is regarded as group $\mathrm{K}$.

\subsection{Participants and Setting. The participants were screened} from the outpatient clinic in the Department of Hepatology in Longhua Hospital. 30 healthy volunteers from Longhua Hospital attached to Shanghai University of TCM were selected as normal control group.

The inclusion criteria of treatment group included the following: (a) Chinese male or female aged from 18 to 60 years old, (b) having the diagnostic criteria of HBeAg negative $\mathrm{CHB}$, according to Chinese Medicine Association "Chronic Hepatitis B Control Guideline, 2011 version," and (c) meeting the syndrome differentiation standards of spleen deficiency and related syndromes (e.g., liver stagnation, spleen deficiency and dampness heat, and spleen deficiency and kidney deficiency) as listed below in detail. The exclusion criteria of treatment group included having not taken antiretroviral therapy and immune therapy within six months and having not received any other treatment within one month.

2.2.1. Criteria of TCM Syndrome. The guiding principles of clinical study of new TCM medicine and the TCM syndrome classification standards in the fifth edition of TCM diagnostics were applied as our criteria of TCM of SD syndrome $[12,13]$. Anyone who has two of the following symptoms: anorexia, fatigue, abdominal distention after eating, and abnormal stool, is diagnosed as having spleen deficiency syndrome. The subtypes of SD syndromes were listed as follows. (a) SDLD meets the diagnosis of spleen deficiency syndrome and has any three of the following symptoms: chest and hypochondrium; abdominal distention and pain; belching; acid regurgitation; depression or irritability; frequent sighing; dry and bitter mouth or obstruction in throat; and so on. (b) SDDH meets the diagnosis of spleen deficiency syndrome and has any three symptoms of thirst but no desire of water; loose stools; heavy limbs; dull fever; red tongue and slimy yellow fur; pulse and moist; and so on, so it is diagnosed. (c) SDKD meets the diagnosis of spleen deficiency syndrome and has any two symptoms of soreness and weakness of waist and knees; sexual hypoactivity; extreme chilliness; frequent urination at night; dysphoria in chestpalms-soles, so it is diagnosed. Every syndrome is recorded as 0, 1, 2, and 3, according to the severity. Two senior TCM professional physicians evaluated the syndrome independently, and only the consistent results were included.

The study was approved by the Institute Review Board of Shanghai University of TCM. All participants were given a written informed consent form and were free to withdraw from the trial at any time.

2.3. Interventions. Patients in group A were given ChaihuShugan in combination with Sijunzi decoction (Codonopsis pilosula $10 \mathrm{~g}$, Atractylodes macrocephala Koidz. $9 \mathrm{~g}$, Poria cocos $9 \mathrm{~g}$, Glycyrrhiza uralensis $6 \mathrm{~g}$, Bupleurum chinense $6 \mathrm{~g}$, Pericarpium citri Reticulatae $6 \mathrm{~g}$, Rhizoma Chuanxiong $4.5 \mathrm{~g}$, Rhizoma Cyperi 4.5 g, Fructus Aurantii $4.5 \mathrm{~g}$, and Paeonia lactiflora $4.5 \mathrm{~g}$ ); patients in group B were given Yinchenhao decoction in combination with Sijunzi decoction (Codonopsis pilosula $10 \mathrm{~g}$, Atractylodes macrocephala Koidz. 9g, Poria cocos $9 \mathrm{~g}$, Glycyrrhiza uralensis 6g, Artemisia capillaris Thunb. 30 g, Gardenia jasminoides $15 \mathrm{~g}$, and Rheum palmatum $9 \mathrm{~g}$ ); patients in group C are given Liuwei Dihuang Wan or Jingui Dihuang Wanin combination with Sijunzi decoction (Codonopsis pilosula $10 \mathrm{~g}$, Atractylodes macrocephala Koidz. $9 \mathrm{~g}$, Poria cocos $9 \mathrm{~g}$, Glycyrrhiza uralensis $6 \mathrm{~g}$, Rehmannia glutinosa $24 \mathrm{~g}$, fructus corni $12 \mathrm{~g}$, Dioscorea opposita $12 \mathrm{~g}$, Rhizoma Alismatis 9g, Cortex Moutan 9g, Ramulus Cinnamomi $9 \mathrm{~g}$, and Aconitum carmichaeli Debx $3 \mathrm{~g}$ ) (Table 1). The decoction was prepared by manufacturing laboratory in Longhua Hospital attached to Shanghai University of 
TABLE 1: Composition of the formula.

\begin{tabular}{|c|c|c|c|}
\hline Group & $\begin{array}{c}\text { SD } \\
\text { category }\end{array}$ & Decoction name & Chinese herbs \\
\hline A & SDLD & $\begin{array}{l}\text { ChaihuShugan and } \\
\text { Sijunzi decoction }\end{array}$ & $\begin{array}{l}\text { Codonops is pilosula } 10 \mathrm{~g} \text {, Atractylodes } \\
\text { macrocephala Koidz. } 9 \mathrm{~g}, \\
\text { Poria cocos } 9 \mathrm{~g}, \\
\text { Glycyrrhiza uralensis } 6 \mathrm{~g} \text {, Bupleurum } \\
\text { chinense } 6 \mathrm{~g} \text {, Pericarpium citri Reticulatae } \\
6 \mathrm{~g} \text {, } \\
\text { Rhizoma Chuanxiong } 4.5 \mathrm{~g} \text {, Rhizoma } \\
\text { Cyperi } 4.5 \mathrm{~g}, \\
\text { Fructus Aurantii } 4.5 \mathrm{~g}, \\
\text { Paeonia lactiflora } 4.5 \mathrm{~g}\end{array}$ \\
\hline B & SDDH & $\begin{array}{l}\text { Yinchenhao and } \\
\text { Sijunzi decoction }\end{array}$ & $\begin{array}{l}\text { Codonops is pilosula } 10 \mathrm{~g} \text {, Atractylodes } \\
\text { macrocephala Koidz. } 9 \mathrm{~g} \text {, } \\
\text { Poria cocos } 9 \mathrm{~g}, \\
\text { Glycyrrhiza uralensis } 6 \mathrm{~g} \text {, Artemisia } \\
\text { capillaris Thunb. } 30 \mathrm{~g}, \\
\text { Gardenia jasminoides } 15 \mathrm{~g} \text {, Rheum } \\
\text { palmatum } 9 \mathrm{~g}\end{array}$ \\
\hline $\mathrm{C}$ & SDKD & $\begin{array}{l}\text { Liuwei Dihuang } \\
\text { Wan or Jingui } \\
\text { Dihuang Wanin } \\
\text { combination with } \\
\text { Sijunzi decoction }\end{array}$ & $\begin{array}{l}\text { Codonopsispilosula } 10 \mathrm{~g}, \\
\text { Atractylodes macrocephala Koidz. } 9 \mathrm{~g}, \\
\text { Poriacocos } 9 \mathrm{~g}, \text { Glycyrrhiza uralensis } 6 \mathrm{~g}, \\
\text { Rehmannia glutinosa } 24 \mathrm{~g}, \\
\text { fructus corni } 12 \mathrm{~g}, \text { Dioscorea opposita } 12 \mathrm{~g} \text {, } \\
\text { Rhizoma Alismatis } 9 \mathrm{~g} \text {, Cortex Moutan } \\
9 \mathrm{~g}, \\
\text { Ramulus Cinnamomi } 9 \mathrm{~g}, \\
\text { Aconitum carmichaeli Debx } 3 \mathrm{~g}\end{array}$ \\
\hline
\end{tabular}

TCM in vacuum bags $(150 \mathrm{~mL} / \mathrm{bag})$ and the patients were instructed to take the medicine $(150 \mathrm{~mL})$ twice a day for each prescription, and the duration of one-course treatment was three months.

\subsection{Outcomes}

2.4.1. TCM Syndrome Score Calculation. The scores are recorded and marked as obviously effective: symptoms and signs are improved markedly, and symptom scores decrease $\geq 70 \%$; effective: symptoms and signs are improved gradually, and symptom scores decrease $\geq 30 \%$; invalid: symptoms and signs are not significantly improved, and symptom scores decrease $\leq 30 \%$. The formula (nimodipine method) is as follows: [(total score before treatment - total score after treatment)/total score before treatment] $\times 100 \%[14]$.

2.4.2. Reagents and Instruments. RPMI-1640 culture medium and fetal bovine serum were purchased from GIBCO (USA); granulocyte-macrophage colony stimulating factor (GMCSF), recombinant human interleukin-4 (IL-4), and tumor necrosis factor- $\alpha$ (TNF- $\alpha$ ) were obtained from Peprotech (USA); human interleukin-10 (IL-10), human interleukin-12 (IL-12), and ELISA kit were from R\&D company (USA); fluorescence labeling CD80-FITC, CD86-PE, CD1a-PECY5, and HLA-DR-APC monoclonal antibodies were from $\mathrm{BD}$ Company (USA); FACSCalibur flow cytometry was also from BD Company (USA).
2.4.3. The Liver Function and $H B V$-DNA Detection. Liver function detection applied biochemical rate method and was done by clinical laboratory in Longhua Hospital attached to Shanghai University of TCM. HBV-DNA was detected by fluorescent quantization and implemented by Shanghai Aidicang Medical Diagnostic Centre.

2.4.4. Separation of Peripheral Blood Mononuclear Cell (PBMC). The method was applied according to the literature $[15,16]$. In brief, $10 \mathrm{~mL}$ peripheral blood is collected from each subject, under sterile conditions; after the heparin anticoagulation, same volume of PBS is added for dilution. Spread the mixture on the lymphocyte separation medium, centrifuge $800 \mathrm{~g} / \mathrm{min}$ for $20 \mathrm{~min}$ at room temperature, collect the cells in the interface between upper and middle layer, add PBS for suspension and washing, add serum-free RPMI1640 medium to precipitate the cells, count and adjust the cell concentration to $2 \sim 6 \times 10^{6} / \mathrm{mL}$, add $1.5 \mathrm{~mL}$ for each group, put the cells into plates, incubate the cells for 2 hours in an incubator at $37^{\circ} \mathrm{C}$ with a humidified $5 \% \mathrm{CO}_{2}$ atmosphere, remove nonadherent cells, and then get the peripheral blood mononuclear cells.

2.4.5. Differentiation and Culture for DCs In Vitro. The method was applied according to the literature [17-19]. Wash mononuclear cells with serum-free RPMI 1640 for three times, add 10\% fetal bovine serum RPMI 1640 medium, and add cytokine into the final concentration of $100 \mathrm{ng} / \mathrm{mL}$ 
TABLE 2: The comparison of gender, age, and course of disease of HBeAg negative CHB patients at baseline.

\begin{tabular}{|c|c|c|c|c|}
\hline \multirow{2}{*}{ Group } & \multicolumn{2}{|c|}{ Gender $(n)$} & \multirow{2}{*}{ Age (year) } & \multirow{2}{*}{ Course (year) } \\
\hline & Male & Female & & \\
\hline $\bar{A}$ & 18 & 12 & $38.15 \pm 6.18^{*}$ & $8.56 \pm 3.45$ \\
\hline B & 17 & 13 & $37.65 \pm 7.09^{*}$ & $9.43 \pm 4.38$ \\
\hline $\mathrm{C}$ & 16 & 14 & $46.35 \pm 10.34$ & $11.73 \pm 4.43$ \\
\hline
\end{tabular}

TABLE 3: The comparison of liver enzymes and HBV-DNA levels in HBeAg negative CHB patients before and after treatment.

\begin{tabular}{|c|c|c|c|c|}
\hline Group & ALT (U/L) & $\operatorname{AST}(\mathrm{U} / \mathrm{L})$ & TBIL (umol/L) & HBV-DNA (IU/ML) \\
\hline \multicolumn{5}{|l|}{ A } \\
\hline Before & $85.34 \pm 15.14$ & $71.26 \pm 22.34$ & $14.68 \pm 4.34$ & $2.23 E+7 \pm 4.35 E+5$ \\
\hline After & $50.36 \pm 8.53^{\#}$ & $43.78 \pm 11.25^{\#}$ & $13.53 \pm 6.29$ & $1.98 E+7 \pm 2.97 E+6$ \\
\hline \multicolumn{5}{|l|}{ B } \\
\hline Before & $91.06 \pm 16.20$ & $77.13 \pm 17.21$ & $17.54 \pm 6.85$ & $1.47 E+7 \pm 3.96 E+5$ \\
\hline After & $54.67 \pm 9.15^{\#}$ & $48.56 \pm 13.21^{\#}$ & $15.83 \pm 7.61$ & $4.32 E+6 \pm 1.24 E+5^{*}$ \\
\hline \multicolumn{5}{|l|}{$\mathrm{C}$} \\
\hline Before & $83.94 \pm 17.53$ & $69.38 \pm 23.16$ & $18.65 \pm 5.38$ & $2.30 E+7 \pm 4.63 E+5$ \\
\hline After & $49.86 \pm 7.33^{\#}$ & $39.66 \pm 9.64^{\#}$ & $14.79 \pm 8.35$ & $8.73 E+6 \pm 4.16 E+5^{*}$ \\
\hline
\end{tabular}

${ }^{*} P<0.05$, compared to group $C ;{ }^{\#} P<0.05$, compared to baseline.

and rhIL-4 $100 \mathrm{ng} / \mathrm{mL}$ of rhGM-CSF. Continue to cultivate the cells and change the medium every $48 \mathrm{~h}$ (including the cytokine, rhGM-CSF $50 \mathrm{ng} / \mathrm{mL}$, rhIL-4 $50 \mathrm{ng} / \mathrm{mL}$ ), and then add TNF- $\alpha(100 \mathrm{ng} / \mathrm{mL})$ on the fifth day, incubate 48 hours, and collect DCs.

2.4.6. DCs Morphology and Proliferation Observation. Observe DCs morphology and proliferation under phasecontrast microscope on the first, third, fifth, and seventh day and observe the morphology of mature DCs with scanning electron microscope.

2.4.7. Detection of DCs Surface Markers. Cultivate the DCs for seven days and detect the surface molecules of DCs, such as CD80, CD86, and CDla, and the expression level of HLADR with flow cytometry. All the procedures were conducted according to the kits [18].

2.4.8. Detection of $I L-10$ and $I L-12$. Collect the DCs cell supernatant and detect the content of cytokines IL-10 and IL12 with ELISA method.

2.5. Statistical Methods. Measurement data is represented by mean \pm standard deviation (SD). HBV-DNA was compared after logarithmic transformation. Use variance analysis method and least significant difference (LSD) to compare the difference between groups and use paired $t$-test to compare the mean of each group before and after the treatment. All the data were analyzed by SPSS 18.0 software and a $P$ value less than 0.05 was considered statistically significant.

\section{Results}

3.1. Baseline Data. 114 patients diagnosed with "spleen deficiency" were included, and 24 participants were excluded after syndrome screening. $90 \mathrm{HBeAg}$ negative $\mathrm{CHB}$ patients were recruited from the outpatient clinic in the Department of Hepatology in Longhua Hospital from January 2012 to December 2012: 51 males (age range from 19 to 60 years; average age is 39.5 years), with 2-21 years disease course (average course is 9.8 years), and 39 females (age range from 20 to 60 years; average age is 44.3 years), with $2-20$ years disease course (average course is 10.6 years). The comparative difference between gender, course of disease, liver function, and HBV-DNA levels of $\mathrm{HBeAg}$ negative $\mathrm{CHB}$ patients in three different spleen deficiency syndrome groups before treatment has no statistical significance $(P>0.05)$. The age of patients in group $C$ was higher than that of group $A$ and group B $(P<0.05)$ (Tables 2 and 3 , Figure 1$)$.

3.2. The Effects of Chinese Medicine Treatment according to Syndrome Differentiation among HBeAg Negative CHB. Compared with baseline, all the enzymes, reflecting liver function and ALT and AST levels in each group, decreased significantly $(P<0.05)$ (Table 3 , Figures $1(\mathrm{a})$ and $1(\mathrm{~b})$ ) while the total bilirubin (TBIL) among groups had no significant difference (Table 3, Figure 1(c)). Similarly, HBVDNA in group $\mathrm{B}$ and group $\mathrm{C}$ reduced significantly compared to that before treatment $(P<0.05)$ (Table 3, Figure 1(e)). The total syndrome scores in each group also improved significantly after treatment $(P<0.05)$. In group $\mathrm{A}$, there were 10 obviously effective cases, 9 effective cases, and 1 


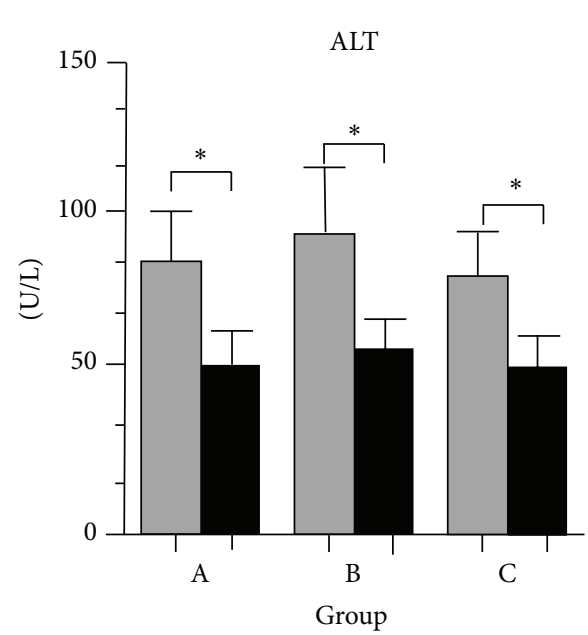

(a)

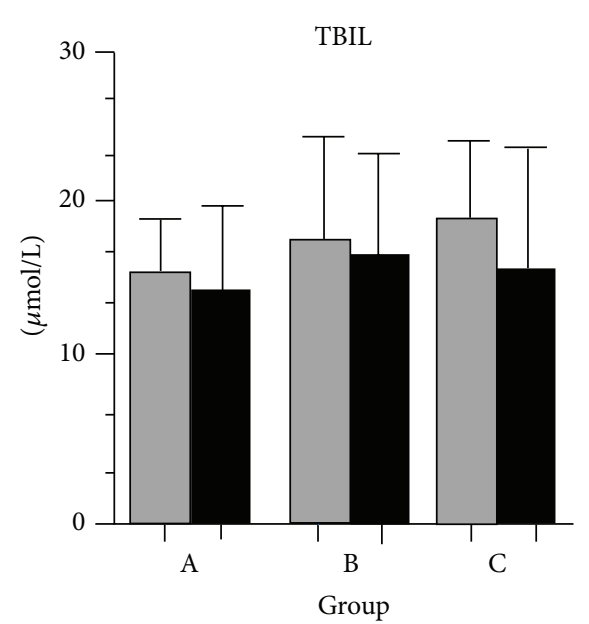

(c)

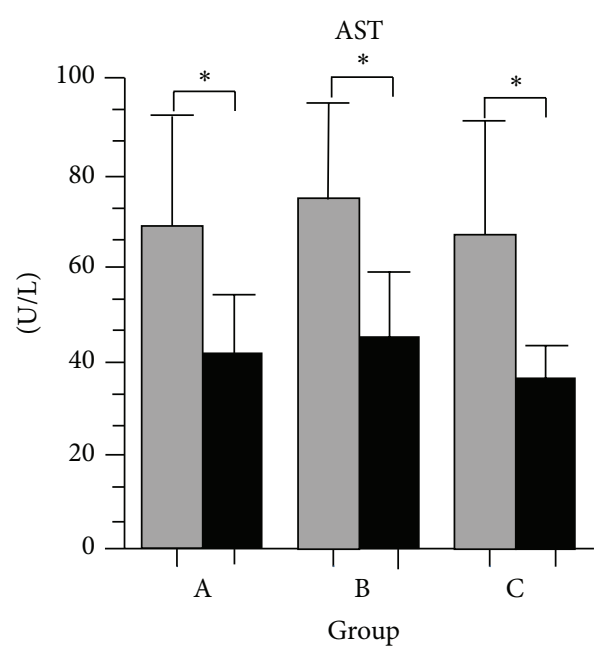

(b)

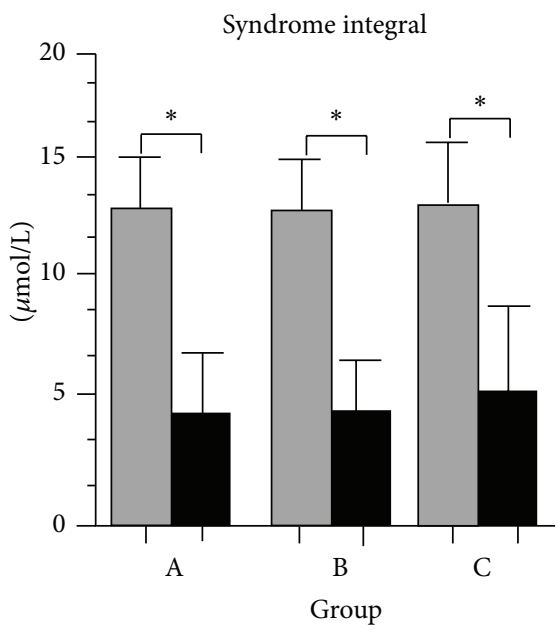

(d)

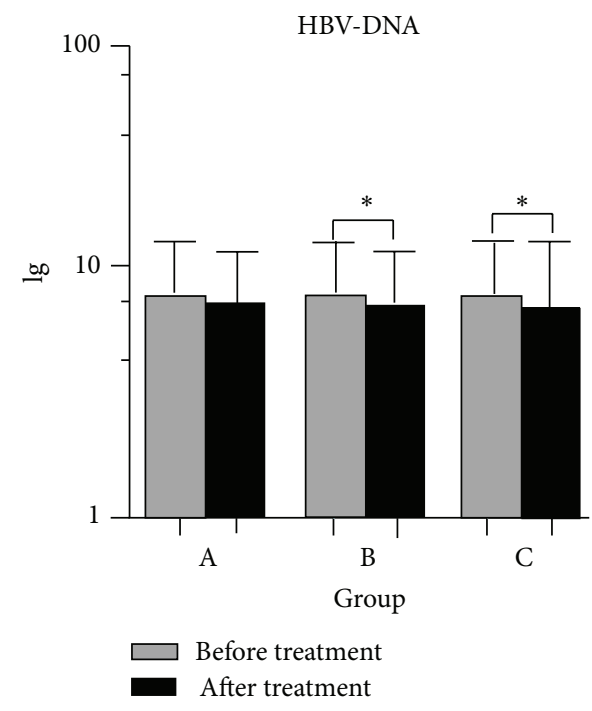

(e)

FIGURE 1: The effectiveness of treatment according to syndrome differentiation among HBeAg negative CHB patients. (a) Comparison of ALT levels before and after treatment in each group. (b) Comparison of AST levels before and after treatment in each group. (c) Comparison of TBIL levels before and after treatment in each group. (d) Comparison of syndrome scores before and after treatment in each group. (e) Comparison of HBV-DNA load before and after treatment in each group. 30 patients in each group; ${ }^{*}$ indicates $P<0.05$ between groups. 


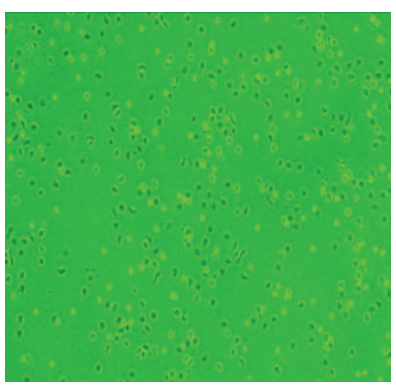

(a)

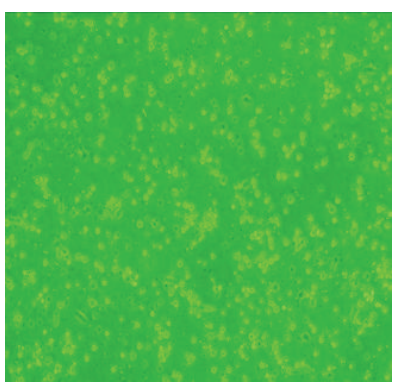

(b)

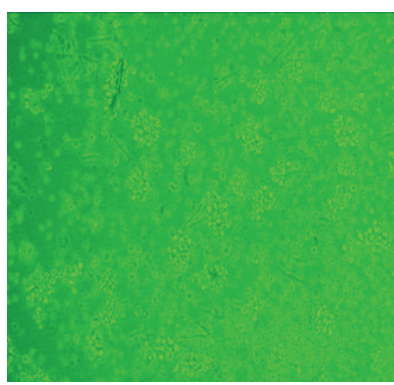

(c)

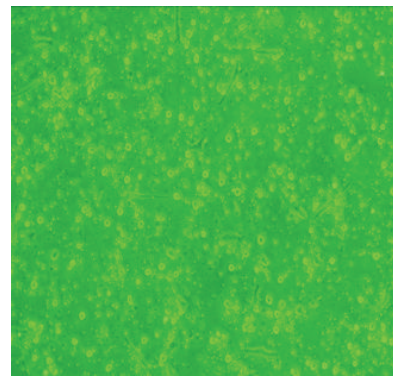

(d)

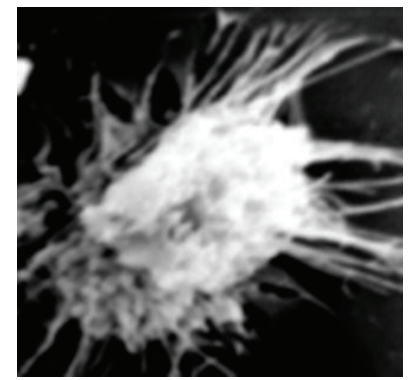

(e)

Figure 2: Morphological changes of DCs during different periods. (a) The second hour of culture. (b) The third day of culture. (c) On the fifth day of culture. (d) On the seventh day of culture. (e) On the seventh day, the DCs morphology was observed by scanning electron microscope.

TABLE 4: The comparison of DCs biomarkers, IL-10, and IL-12 levels in HBeAg negative CHB patients before and after treatment.

\begin{tabular}{|c|c|c|c|c|c|c|}
\hline Group & CD80 (\%) & CD86 (\%) & CDla (\%) & HLA-DR (\%) & IL-10 (pg/mL) & IL-12 (pg/mL) \\
\hline K & $65.03 \pm 9.25$ & $75.60 \pm 7.26$ & $63.21 \pm 15.43$ & $59.21 \pm 15.14$ & $14.31 \pm 6.22$ & $36.67 \pm 8.23$ \\
\hline \multicolumn{7}{|l|}{ A } \\
\hline Before & $12.04 \pm 10.54^{\# \& 8}$ & $49.51 \pm 13.35^{\#}$ & $32.37 \pm 10.06^{\# \&}$ & $52.32 \pm 16.01^{\&}$ & $47.43 \pm 24.59^{\# \&}$ & $16.25 \pm 6.12^{\# \&}$ \\
\hline After & $35.20 \pm 10.47^{*}$ & $64.08 \pm 11.21^{*}$ & $45.15 \pm 11.31^{*}$ & $53.83 \pm 12.99$ & $24.88 \pm 12.06^{*}$ & $26.12 \pm 9.32^{*}$ \\
\hline \multicolumn{7}{|l|}{ B } \\
\hline Before & $9.13 \pm 6.95^{\#}$ & $51.38 \pm 13.74^{\#}$ & $30.11 \pm 10.58^{\# \&}$ & $43.26 \pm 16.35^{\#}$ & $51.43 \pm 24.41^{\#}$ & $14.49 \pm 5.03^{\# 8}$ \\
\hline After & $42.66 \pm 13.09^{\mathbf{\Delta} *}$ & $68.14 \pm 15.97^{*}$ & $44.52 \pm 10.63^{*}$ & $57.34 \pm 10.68^{*}$ & $23.13 \pm 11.40^{*}$ & $25.49 \pm 8.42^{*}$ \\
\hline \multicolumn{7}{|l|}{$\mathrm{C}$} \\
\hline Before & $6.01 \pm 3.32^{\#}$ & $43.54 \pm 20.06^{\#}$ & $20.24 \pm 9.37^{\#}$ & $38.20 \pm 14.12^{\#}$ & $65.54 \pm 27.18^{\#}$ & $8.69 \pm 4.13^{\#}$ \\
\hline After & $28.46 \pm 7.90^{*}$ & $70.86 \pm 18.42^{*}$ & $38.43 \pm 12.19^{*}$ & $55.81 \pm 13.88^{*}$ & $28.56 \pm 9.78^{*}$ & $20.54 \pm 7.47^{*}$ \\
\hline
\end{tabular}

${ }^{\#} P<0.01$, compared to group K before treatment; ${ }^{\&} P<0.05$, compared to group C before treatment; ${ }^{*} P<0.05$, compared to before treatment in each group; ${ }^{\Delta} P<0.05$, compared to group $\mathrm{C}$ after treatment.

invalid case. In group B, there were 7 obviously effective cases, 12 effective cases, and 1 invalid case. In group C, there were 8 obviously effective cases, 12 effective cases, and there was no significant difference regarding the therapeutic effect among groups (Table 3, Figure 1(d)).

3.3. DCs Morphology and Proliferation. The isolated PBMC displayed as quasicircular adherent mononuclear cells after $2 \mathrm{~h}$ still standing (Figure 2(a)). After 3-day coculture with cytokines, the suspended cells increased and adherent mononuclear cells grew aggregately (Figure 2(b)). Until the fifth day, the size of the cells increased and shaped into a light irregular appearance and the colony presented (Figure 2(c)). On the seventh day, the cells were suspended but could not attach the container; their shape was irregular and numerous extended burrs could be seen. From the further observation by scanning electron microscope, it represented the shape of mature DCs (Figures 2(d) and 2(e)). The DCs proliferation from $\mathrm{CHB}$ patients was relatively slower than that from healthy people.

3.4. The DC Phenotype and the Comparison of IL-10 and IL12 of HBeAg Negative CHB Patients before and after Treatment. Before treatment, the expression rate of DCs surface biomarkers of $\mathrm{HBeAg}$ negative $\mathrm{CHB}$ patients was significantly lower than healthy people $(P<0.05)$ and the expression level of DCs surface biomarkers between various syndrome types was different (Table 4). Specifically, the positive expression rate of CD80, CD1a, and HLA-DR in group A patients was higher than that of group $C(P<0.05)$, and 
the positive expression rate of CD1a in group B patients was higher than that of group $\mathrm{C}(P<0.05)$. Moreover, the content of IL-10 in DCs supernatant of CHB patients was higher than healthy people, while the IL-12 content is lower $(P<0.05)$. IL-10 content in group $\mathrm{C}$ is significantly higher than that of group A, while IL-12 content is lower than that of group $\mathrm{A}$ and group $\mathrm{B}(P<0.05)$. Compared to the baseline, the expression of CD80 (Table 4, Figure 3(a)), CD86 (Table 4, Figure 3(b)), CDla (Table 4, Figure 3(c)), HLA-DR (Table 4, Figure 3(d)), and IL-12 (Table 4, Figure 3(f)) content after the treatment in each group significantly increased $(P<0.05)$, while IL-10 content decreased (Table 4, Figure 3(e)). After the treatment, the comparison of other surface markers of DCs has no statistical significance (Table 4, Figure 3), except that the expression of CD80 in group B is higher than that of group C.

\section{Discussion}

The HBV infection is a public health problem attracting global attention. The continuous infection mechanism of $\mathrm{HBV}$ is not completely defined, and the defect of host immune is regarded as an important mechanism [20]. DCs are the most potent antigen presenting cells, and studies showed that chronic HBV infected patients have the defect of DCs maturity and function, so it cannot deliver the signal of virus antigens to the immune system, which is one of the major mechanisms of chronicity of hepatitis B $[21,22]$. Generally the differentiation and development of DCs go through two stages. Immature DCs have the ability of capturing and processing antigens, after taking in the antigens; they can spontaneously mature and obtain the ability of activating primary type $\mathrm{T}$ cells and complete immune stimulating function [23]. Compared with immature DCs, the antigen capture ability of mature DCs decreases, but their antigen presenting ability is obviously enhanced. Previous research has shown that the decreased number, immature phenotype, and defected function of DCs of peripheral blood in $\mathrm{CHB}$ patients caused the failure of antigens presenting to CD4+ adjuvanticity T lymphocytes (Th) and CD8+ cytotoxic T lymphocytes (CTLs) [24, 25].

Traditional Chinese medicine plays an indispensable role in CHB and even HCC treatment [26]. Not only is it an effective treatment method for patients who do not need antiviral therapy but also it can improve the immune function and have antiviral effect according to some research findings [27]. Based on long-term clinical practice and pilot observational study, our research team found that $\mathrm{HBeAg}$ negative $\mathrm{CHB}$ patients had a certain amount of "spleen deficiency" syndrome and also the syndrome of "liver depression," "damp heat," "kidney deficiency," and so on. This different syndrome classification based on "spleen deficiency" had differences of basic immune level and medical treatment sensitivity. This clinical research, through observing the changes of patients' syndrome scores before and after treatment, evaluating the effectiveness of "syndrome differentiation" objectively, and illustrating the scientific mechanisms of different Chinese syndromes based on the research hypothesis of DC functional state, provided referable evidence for TCM in treating
$\mathrm{HBeAg}$ negative $\mathrm{CHB}$ patients and helped the investigators to determine the appropriate therapies for $\mathrm{HBeAg}$ negative $\mathrm{CHB}$ patients based on the different Chinese medicine syndromes according to evidence-based medical treatment [28].

Our results also showed that there was no significant difference of gender distribution, course of disease, liver function, and HBV-DNA load before treatment among three different "spleen deficiency" syndromes of $\mathrm{CHB}$ patients, while the age of SDKD patients was older than that of SDLD and SDDH patients. This may be explained by TCM theory, where the appearance of "kidney deficiency" is associated with the increase of age and the course of disease is related to the progress of pathology. In our study, we observed that the age in SDKD group was older than that in the other two groups, which was consistent with the TCM theory. Meanwhile, the course of disease among the three groups had no statistic difference; thus, we considered that the diversity of age in our study was not the main factor to explain the difference of DC function and phenotype.

Our data also showed that TCM treatment according to syndrome differentiation could reduce both syndrome scores and ALT, AST levels compared to the baseline, demonstrating preferable therapeutic effect. Unexpectedly, we found that the HBV-DNA load in SDDH group and SDKD group also decreased after TCM treatment. In suppressing HBV-DNA replication, it is usually considered that nucleoside analogues and interferon are superior, while our data backed that proper TCM treatment (according to syndrome differentiation) might be an optional method to improve therapeutic effect in clinical practice.

The change of DC function and phenotype is one of the important immune mechanisms in $\mathrm{HBeAg}$ negative CHB [21]. Our study observed the change of DCs surface biomarkers including CD80, CD86, CD1a, and HLA-DR and secreted cytokines IL-10, IL-12 (Figure 4). Before treatment, DC surface biomarkers expression was different among the three spleen deficiency syndromes in $\mathrm{HBeAg}$ negative $\mathrm{CHB}$ patients; the expression of CD80, CDla, and HLA-DR in SDLD group and the expression of CDla in SDDH group are higher than those in SDKD group, and the IL-10 content of DCs supernatant in SDKD group is higher than that in SDLD group, while IL-12 content is lower than that in SDLD and SDDH groups. IL-10 is an inhibitory cytokine, and it can decrease the antigen presenting function of DCs, through prohibiting the differentiation and maturity of DCs and inhibiting the secretion of IL-12 [29]. Our results demonstrated the differences of biomarkers expression among the three different spleen deficiency syndromes, indicating some scientific mechanism of the classification syndrome in $\mathrm{HBeAg}$ negative $\mathrm{CHB}$ patients. In our study, both SDLD and SDDH belong to the intermingling syndrome (deficiency and excess combination), so the biomarkers have no significant difference. The DC function and phenotype might be insufficient to distinguish the three different spleen deficiency syndromes, and other characteristic biomarkers are needed to further make the classification of syndromes. After syndrome targeting TCM treatment, the maturity and function of DCs were improved; the syndrome scores and HBV-DNA load all reduced. 


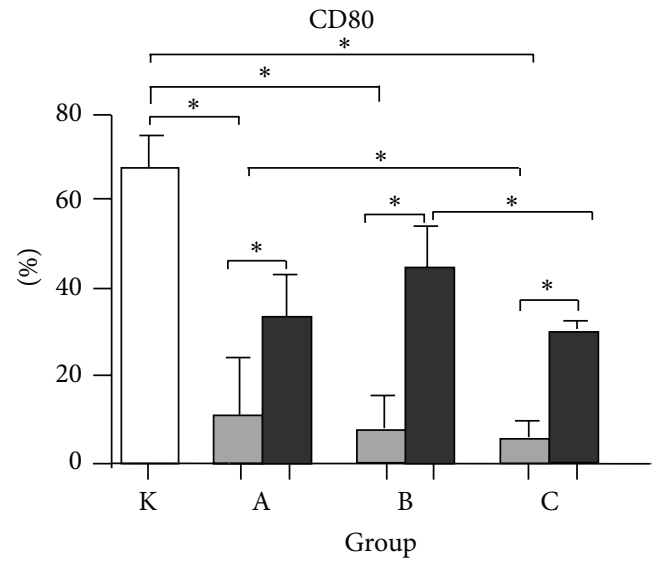

(a)

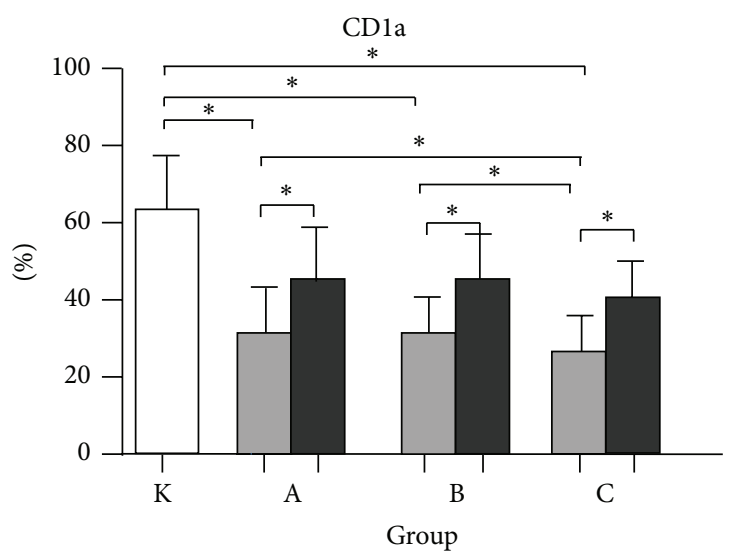

(c)

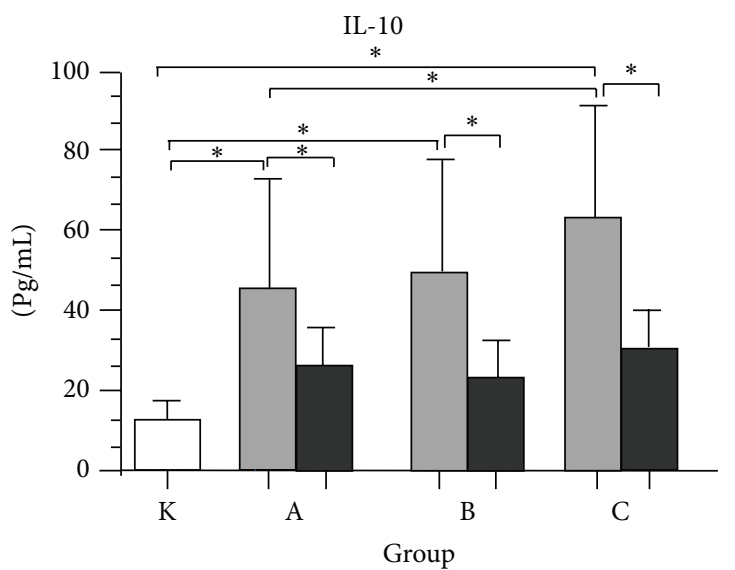

Before treatment After treatment

(e)

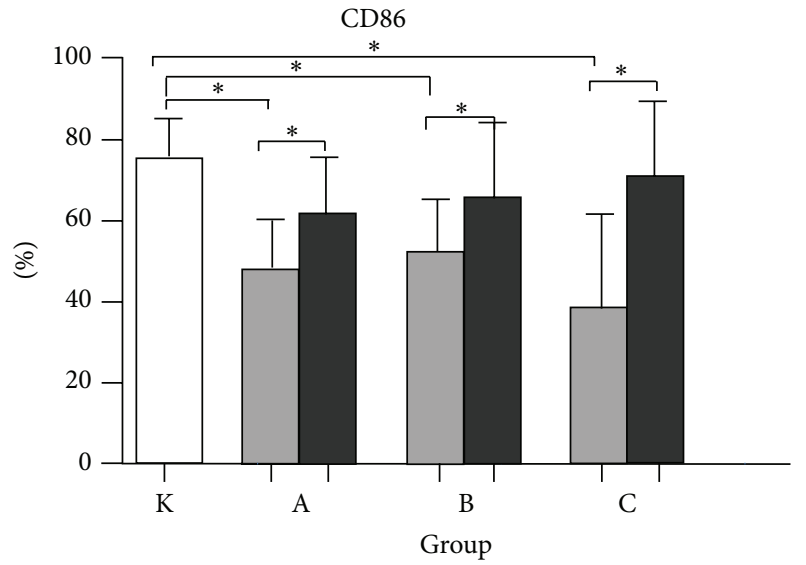

(b)

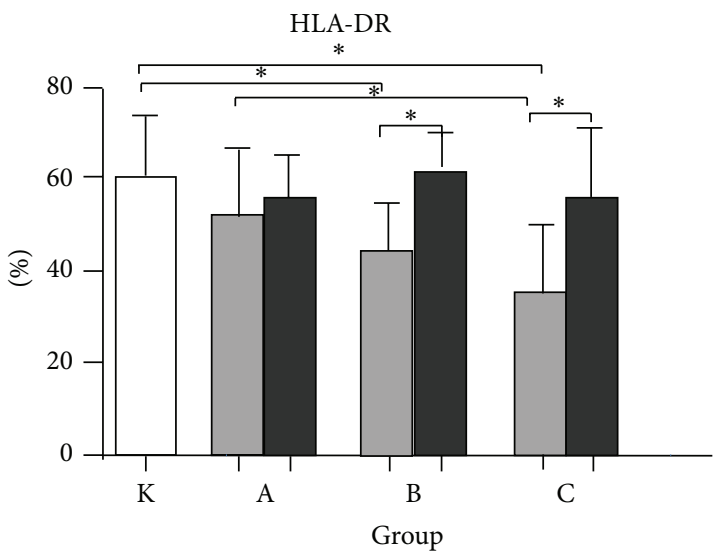

(d)

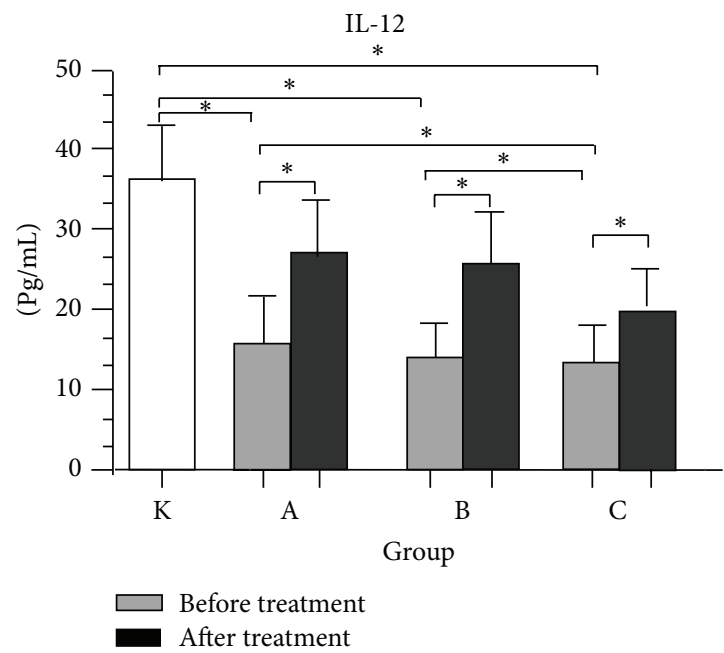

(f)

FIGURE 3: The change of DC phenotype and function of CHB patients in responding to treatment. (a) The comparison of CD80 before and after treatment in each group. (b) The comparison of CD86 before and after treatment in each group. (c) The comparison of CDla before and after treatment in each group. (d) The comparison of HLA-DR before and after treatment in each group. (e) The comparison of IL-10 before and after treatment in each group. (f) The comparison of IL-12 before and after treatment in each group. 30 patient cases in each group; *indicates $P<0.05$ between the corresponding groups. 

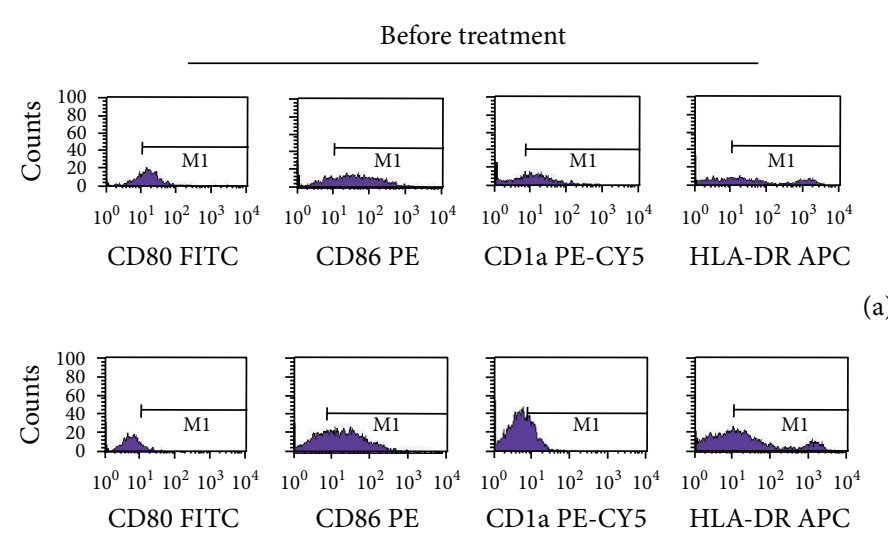

(a)
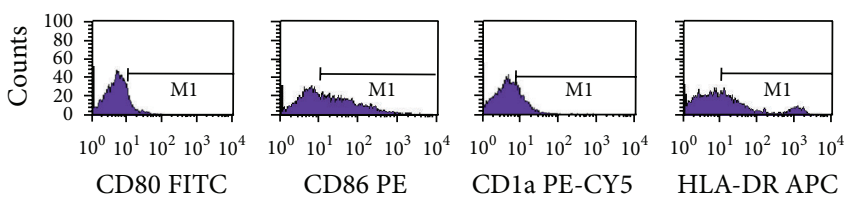

(b)
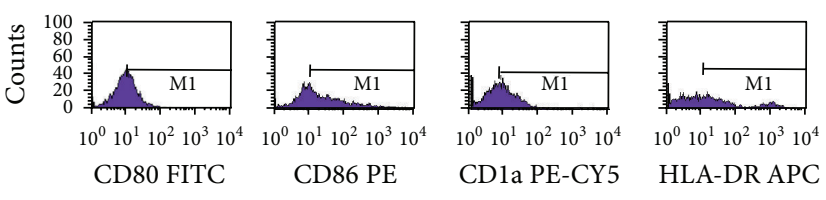

$$
\text { ن }
$$
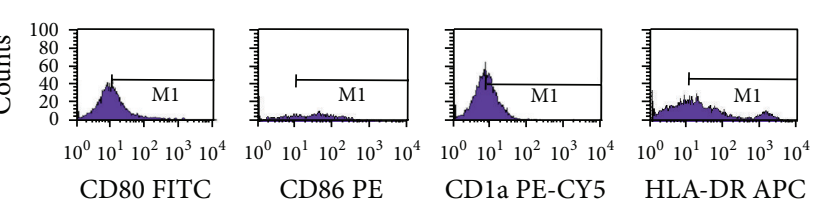

(c)
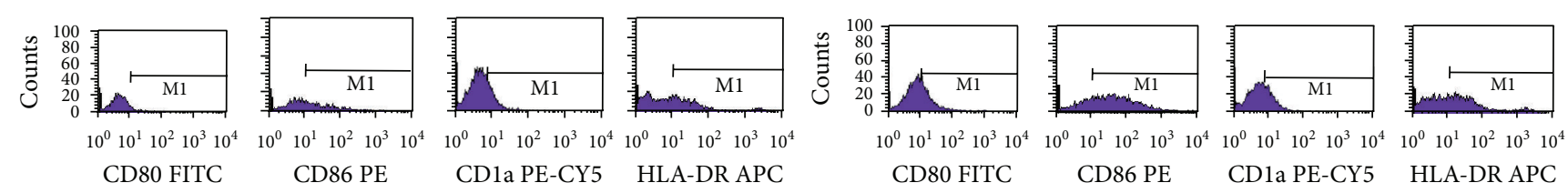

(d)

Figure 4: The change of DCs surface biomarkers before and after treatment in each group: Panel (a) CD80, CD86, CD1a, and HLR-DR expression in DCs from group K; Panel (b) CD80, CD86, CDla, and HLR-DR expression in DCs from group A; Panel (c) CD80, CD86, CD1a, and HLR-DR expression in DCs from group B; Panel (d) CD80, CD86, CDla, and HLR-DR expression in DCs from group C.

Although our findings indicated that Chinese medicine intervention according to syndrome differentiation could advance the maturity and function of DCs as well as other immune indexes, the tentatively positive effects shown in the present study should be interpreted with caution. This study has a number of limitations. Firstly, this is a cohort study without any randomization, which made the self-selection bias obviously present, and the investigators and assessors also may have personal bias in the research outcome measurements. Secondly, this study's sample size is not enough and only 120 participants with 30 in each group which may not be able to represent the real situation of the syndrome distribution and effective size. Thirdly, the quality control of the herbal decoction could not be well controlled as the granules and we would use the high quality control herbal granules in next studies. Fourthly, our study was a small single-centre trial in a single ethnic Chinese population; thus, our findings may not be broadly generalizable.

\section{Conclusion}

Our research showed that TCM therapy based on syndrome differentiation could improve therapeutic effect of $\mathrm{HBeAg}$ negative CHB patients in DC phenotype, liver function, interleukin-10 (IL-10), IL-12, and HBV-DNA levels before and after the treatment. This result might provide some evidence in the rationale of syndrome-based treatments and further large-scale multicenter clinical studies on more diverse population are needed to confirm these findings.

\section{Conflict of Interests}

There is no conflict of interests involved in this paper.

\section{Authors' Contribution}

Lei Wang and Li Zhang contribute equally to this paper.

\section{Acknowledgment}

This work was supported by the National Natural Science Foundation of China (no. 81302891).

\section{References}

[1] G. Brook, V. Soriano, and C. Bergin, "European guideline for the management of hepatitis B and C virus infections, 2010," International Journal of STD and AIDS, vol. 21, no. 10, pp. 669678, 2010.

[2] "The guideline of prevention and treatment for chronic hepatitis B, (2010 version)," Zhonghua Liu Xing Bing Xue Za Zhi, vol. 32, no. 4, pp. 405-415, 2011. 
[3] P. Lan, C. Zhang, Q. Han, J. Zhang, and Z. Tian, “Therapeutic recovery of hepatitis $B$ virus (HBV)-induced hepatocyteintrinsic immune defect reverses systemic adaptive immune tolerance," Hepatology, vol. 58, no. 1, pp. 73-85, 2013.

[4] J. Chang, T. M. Block, and J. Guo, “The innate immune response to hepatitis B virus infection: implications for pathogenesis and therapy," Antiviral Research, vol. 96, no. 3, pp. 405-413, 2012.

[5] E. Segura and S. Amigorena, "Cross-presentation by human dendritic cell subsets," Immunology Letters, vol. 158, no. 1-2, pp. 73-78, 2013.

[6] Y. Bordon, "T cell responses: a dendritic cell designed for two," Nature Reviews Immunology, vol. 13, no. 12, pp. 844-845, 2013.

[7] N. Mathewson, T. Toubai, S. Kapeles et al., "Neddylation plays an important role in the regulation of murine and human dendritic cell function," Blood, vol. 122, no. 12, pp. 2062-2073, 2013.

[8] C. T. Lu, W. H. Zhou, and A. R. Hu, "Study on the relationship between HBV viral loads and the changes of liver pathological features in patients with HBeAg-negative chronic hepatitis B," Zhonghua Shi Yan He Lin Chuang Bing Du Xue Za Zhi, vol. 24, no. 3, pp. 184-186, 2010.

[9] A. Lu, A. Bensoussan, J. Liu, Z. Bian, and W. C. Cho, “TCM Zheng classification and clinical trials," Evidence-Based Complementary and Alternative Medicine, vol. 2013, Article ID 723659, 3 pages, 2013.

[10] C. W. Cheng, S. F. Fu, Q. H. Zhou et al., "Extending the CONSORT Statement to moxibustion," Journal of Chinese Integrative Medicine, vol. 11, no. 1, pp. 54-63, 2013.

[11] F. He, K. W. Sun, and J. P. Peng, "Study on the functions of peripheral dendritic cells in chronic hepatitis B virus infection patients of Gan-depression Pi-deficiency syndrome and GanDan damp-heat syndrome under different immune states," Zhongguo Zhong Xi Yi Jie He Za Zhi, vol. 31, no. 11, pp. 14911495, 2011.

[12] X. Y. Zheng, Guiding Principles of Clinical Research for Traditional Chinese Medicine, The Medicine Science and Technology Press of China, Beijing, China, 2002.

[13] T. T. Deng, Diagnostics of Traditional Chinese Medicine, Shanghai Science and Technology Press, Shanghai, China, 2008.

[14] W. Chen, L. F. Jiang, T. Liu et al., "A clinical observation on spleen-warming network vessels-freeing and orifices-opening formula for 40 cases of Alzheimer's disease," Jounal of Traditional Chinese Medicine, vol. 54, no. 20, pp. 1759-1760.

[15] R. Gupta, S. Kim, and M. W. Taylor, "Suppression of ribosomal protein synthesis and protein translation factors by Peginterferon alpha/ribavirin in HCV patients blood mononuclear cells (PBMC)," Journal of Translational Medicine, vol. 10, article 54, 2012.

[16] M. Averna, R. Stifanese, R. de Tullio et al., "Evidence for alteration of calpain/calpastatin system in PBMC of cystic fibrosis patients," Biochimica et Biophysica Acta-Molecular Basis of Disease, vol. 1812, no. 12, pp. 1649-1657, 2011.

[17] M. Svensson, "Isolation and culture of human hematopoietic progenitors for studies of dendritic cell biology," Methods in Molecular Biology, vol. 531, pp. 187-202, 2009.

[18] E. F. Strasser and R. Eckstein, "Optimization of leukocyte collection and monocyte isolation for dendritic cell culture," Transfusion Medicine Reviews, vol. 24, no. 2, pp. 130-139, 2010.

[19] K. Sugiura, V. Wijewardana, M. Fujimoto et al., "Effect of IL12 on canine dendritic cell maturation following differentiation induced by granulocyte-macrophage CSF and IL-4," Veterinary
Immunology and Immunopathology, vol. 137, no. 3-4, pp. 322326, 2010.

[20] C. P. Desmond, S. Gaudieri, I. R. James et al., "Viral adaptation to host immune responses occurs in chronic hepatitis $\mathrm{B}$ virus (HBV)infection, and adaptation is greatest in HBV e antigennegative disease," Journal of Virology, vol. 86, no. 2, pp. 1181-1192, 2012.

[21] A. M. Woltman, M. L. O. den Brouw, P. J. Biesta, C. C. Shi, and H. L. A. Janssen, "Hepatitis b virus lacks immune activating capacity, but actively inhibits plasmacytoid dendritic cell function," PLoS ONE, vol. 6, no. 1, Article ID e15324, 2011.

[22] M. L. Op Den Brouw, R. S. Binda, M. H. van Roosmalen et al., "Hepatitis B virus surface antigen impairs myeloid dendritic cell function: a possible immune escape mechanism of hepatitis B virus," Immunology, vol. 126, no. 2, pp. 280-289, 2009.

[23] Q. Wang, C. Liu, F. Zhu et al., "Reoxygenation of hypoxiadifferentiated dentritic cells induces Thl and Thl7 cell differentiation," Molecular Immunology, vol. 47, no. 4, pp. 922-931, 2010.

[24] B. J. Zheng, J. Zhou, D. Qu et al., "Selective functional deficit in dendritic cell-T cell interaction is a crucial mechanism in chronic hepatitis B virus infection," Journal of Viral Hepatitis, vol. 11, no. 3, pp. 217-224, 2004.

[25] H. W. Chen, C. H. Liao, C. Ying, C. J. Chang, and C. M. Lin, "Ex vivo expansion of dendritic-cell-activated antigenspecific CD4+ T cells with anti-CD3/CD28, interleukin-7, and interleukin-15: potential for adoptive T cell immunotherapy," Clinical Immunology, vol. 119, no. 1, pp. 21-31, 2006.

[26] X. F. Zhai, Z. Chen, B. Li et al., “Traditional herbal medicine in preventing recurrence after resection of small hepatocellular carcinoma: a multicenter randomized controlled trial," Journal of Chinese Integrative Medicine, vol. 11, no. 2, pp. 90-100, 2013.

[27] H. D. Ma, Y. R. Deng, Z. Tian, and Z. X. Lian, “Traditional Chinese medicine and immune regulation," Clinical Reviews in Allergy and Immunology, vol. 44, no. 3, pp. 229-241, 2013.

[28] A. P. Lv, M. Jiang, and X. R. Ding, "The thought of TCM clinical evaluation research of therapeutic effect based on syndrome classification," Journal of Traditional Chinese Medicine, vol. 50, no. 2, pp. 968-972, 2009.

[29] Y. Chung, N. Zhang, and R. M. Wooten, “Borrelia burgdorferi elicited-IL-10 suppresses the production of inflammatory mediators, phagocytosis, and expression of co-stimulatory receptors by murine macrophages and/or dendritic cells," PLOS ONE, vol. 8, no. 12, Article ID e84980, 2013. 


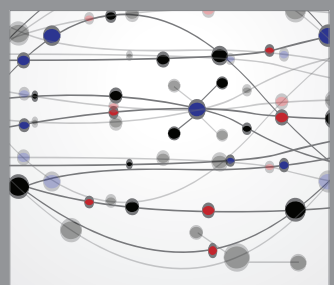

The Scientific World Journal
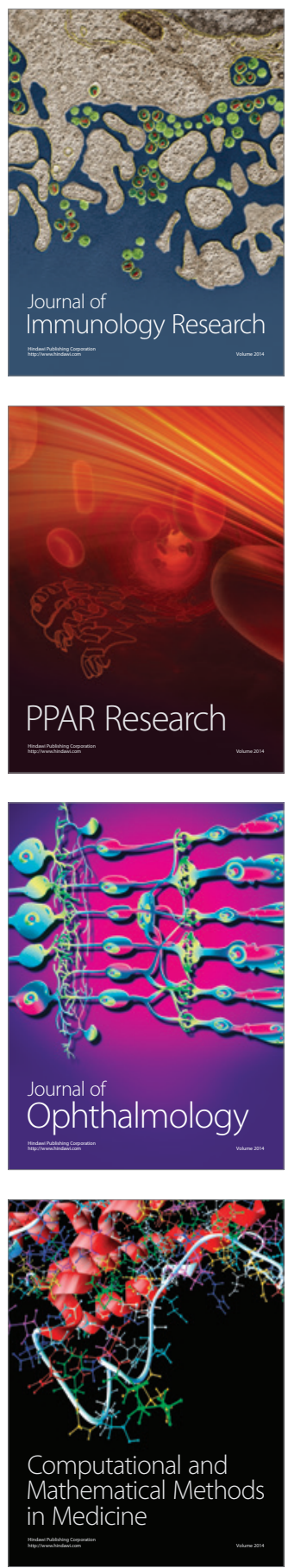

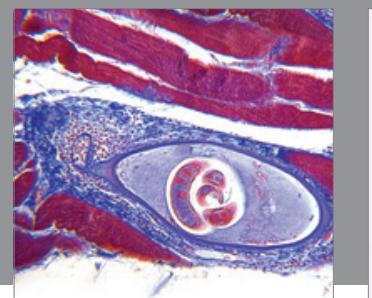

Gastroenterology

Research and Practice
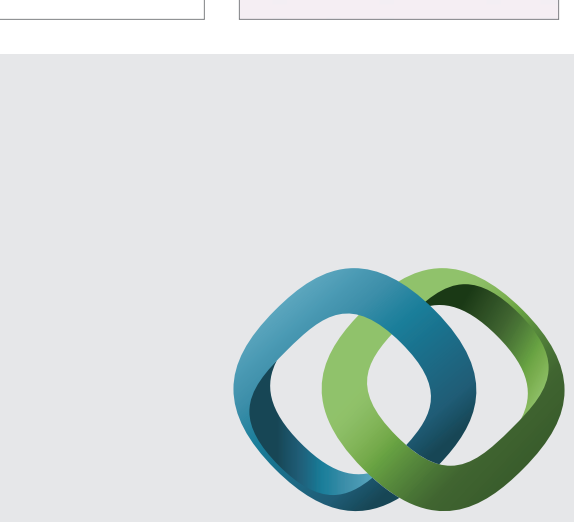

\section{Hindawi}

Submit your manuscripts at

http://www.hindawi.com
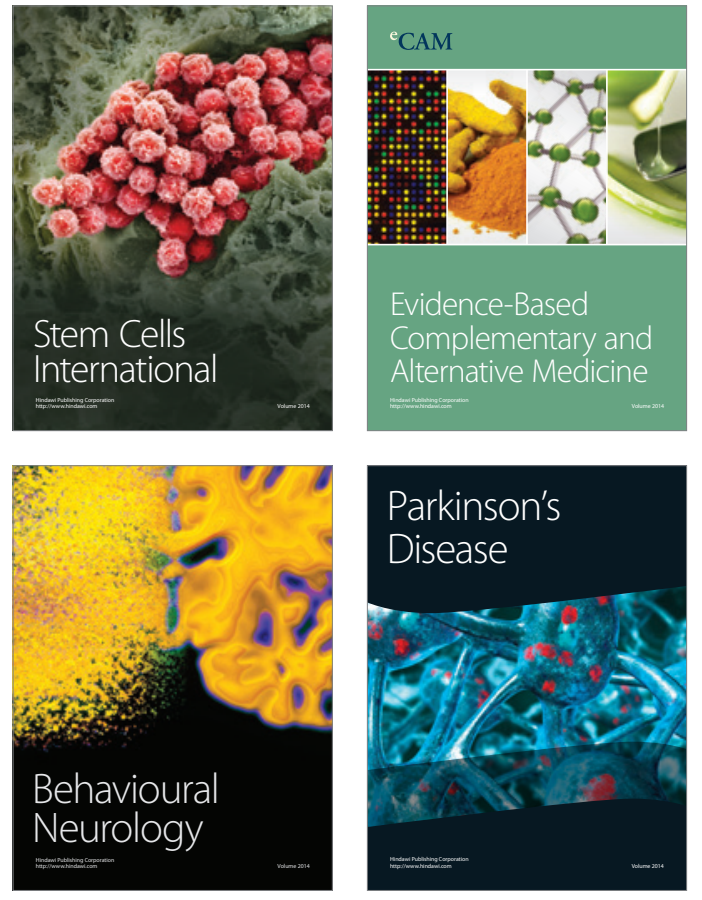
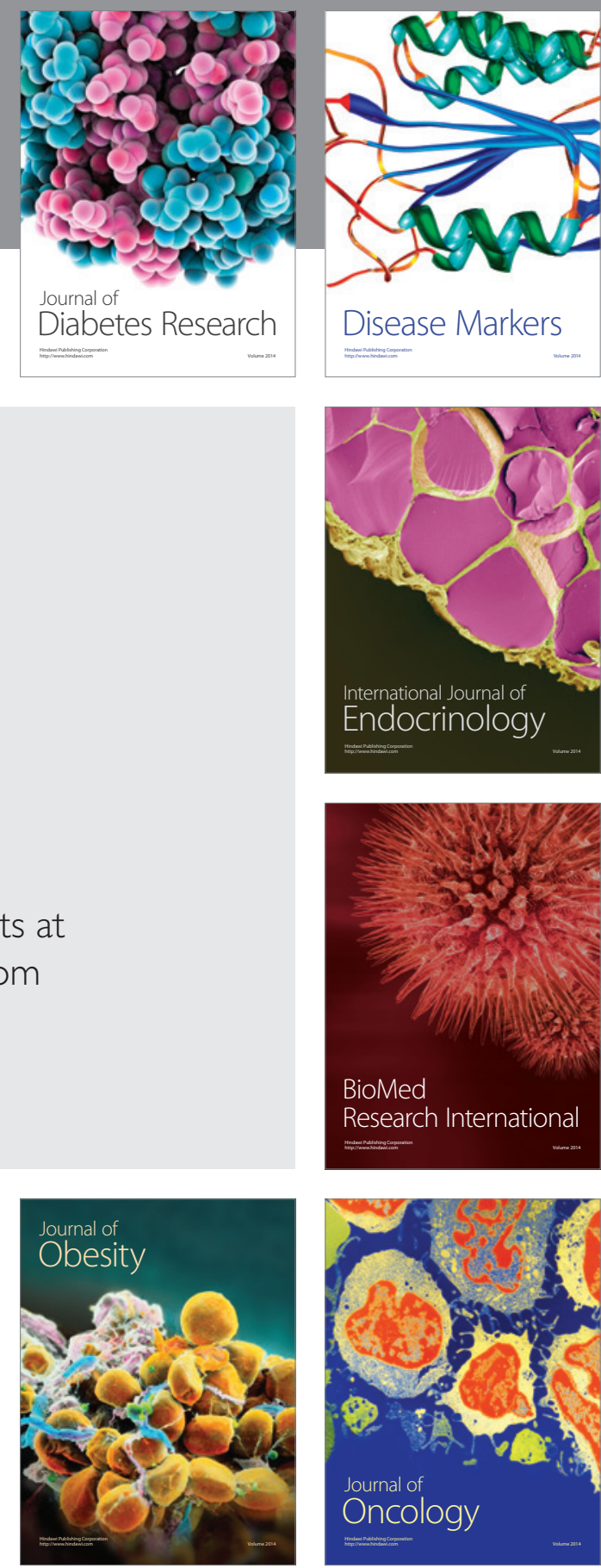

Disease Markers
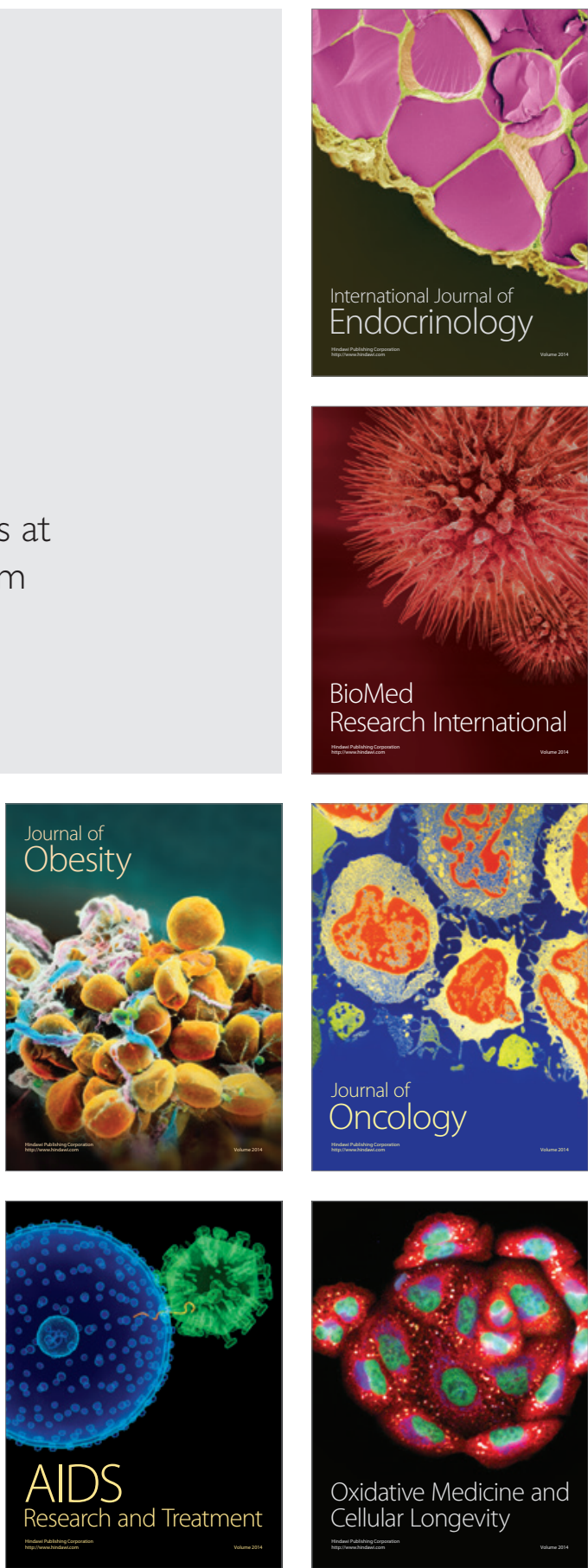